\title{
Fast Browsing of Speech Material for Digital Library and Distance Learning
}

\author{
Peter H.W. Wong* and Oscar C. Au** \\ Department of Electrical and Electronic Engineering \\ The Hong Kong University of Science and Technology \\ Clear Water Bay, Kowloon, Hong Kong. \\ Email: eepeter@ee.ust.hk* eeau@ee.ust.hk**
}

\begin{abstract}
In digital library and distance learning applications, one usually needs to search through lots of speech materials. While content-based retrieval techniques can help to identify possible matching items, the person would usually need to browse through the items quickly before making decisions on whether the items are useful or not. As a result, fast speech browsing techniques are highly desirable.
\end{abstract}

In this paper, we discuss problems of fast playback of speech materials and overview some existing time scale modification (TSM) techniques. We propose some novel modifications of TSM to make it much more effective in fast browsing of speech materials, especially those with irregular speech tempo. The proposed algorithm includes silent period removal, gain equalization and locally adaptive TSM. Simulation results show that the proposed algorithm can increase the intelligibility of the fast playbacked speech materials significantly.

\section{INTRODUCTION}

In recent years, due to the rapid advancement in technologies such as audio and video compression, content-based retrieval and high speed network, digital library and distance learning are quickly becoming feasible possibilities.

In digital library and distance learning applications, two basic needs are the playback of audio and video materials. Such needs arise also in internet access. Even with advance audio/speech compression, audio and speech files in these applications tend to be very large resulting in long downloading time, and network usage. While content-based retrieval techniques can help to identify possible matching items; one usually need to browse through the items quickly before decisions are made as to whether the items are useful or not. It is thus highly desirable to be able to play back audio and speech material at high speed and high quality.

For fast audio and speech playback, something better than simple subsampling must be used because subsampling results in unnatural speeches with shifts in frequency contents. Time scale modification (TSM) is a class of techniques capable of adjusting the apparent speaking rate without affecting the pitch and the frequency contents. In this paper, we propose a novel way of applying time scale modification techniques to process speech material for high quality high speed playback.

\section{TIME SCALE MODIFICATION}

There are at least two major approaches to time scale modification (TSM):

1. Least square error estimates [1] which is a recursive technique to estimate a rate changed signal by minimizing, in mean square sense, the Euclidean distance between the short time Fourier transform (STFT) of the original and rate changed signal.

2. Time frequency models [2] which also use STFT. These models allow time and frequency to be manipulated independently to achieve a speech modification goal. Some well know time domain methods include time domain harmonic scaling (TDHC) [3] and synchronized overlap-add (SOLA) [4].

In this paper, SOLA is used to achieve TSM. In SOLA, the input (or analysis) signal $x[n]$ is segmented into overlapping frames of length $N$ that are a distance of $S_{a}$ apart. The first frame is directly copied to the output (or synthesized) signal $y[n]$. The $(m+1)^{\text {th }}$ input frame which starts at $m S_{a}$ slides along the synthesized signal $y[n]$ around the location $m S_{s}$ in the range of $\left[k_{m i n}, k_{\max }\right]$ to find a location which maximize the normalized cross-correlation function $R(k)$ defined as

$$
R(k)=\frac{\sum_{i=0}^{L-1} y\left[m S_{s}+k+i\right] x\left[x S_{a}+i\right]}{\left\{\sum_{i=0}^{L-1} y^{2}\left[m S_{s}+k+i\right] \sum_{i=0}^{L-1} x^{2}\left[m S_{a}+i\right]\right\}^{1 / 2}}
$$

where $L$ is the length of the overlapping region between 
the shifted analysis frame and the synthesized signal. Once the location with maximum correlation is found, the overlapping region is cross-faded and the remaining of the analysis frame is directly copied to the synthesized signal.

The $S_{a}$ and $S_{s}$ are called analysis and synthesis frame period respectively, and the ratio $\alpha=S_{S} / S_{a}$ is the time scale modification (TSM) factor. When $\alpha$ is less than unity, fast playback is achieved.

While computation requirement for SOLA can be high, fast SOLA is available [5].

\section{MOTIVATION OF ADAPTIVE TSM}

Suppose the target overall fast playback factor or the TSM factor is $\alpha_{0}$. While simple TSM can be applied to the input speech signals directly, we propose to apply locally adaptive TSM due to the following observations on recorded speech, especially those associated with video:

1. when the time scale modification factor $\alpha$ is too small, for example, $\alpha=0.25$, the speech becomes too fast for the untrained human ear to listen.Intelligibility can be very low, if not zero.

2. different people speak with different habitual tempo or speaking rate. This can be due to culture, family background, etc.

3. people speak with variable tempo or speaking rate, sometimes fast and sometimes slow. They tend to speak slowly when they are thinking, hesitating, feeling sad, etc. They tend to speak fast when he know exactly what they want to say, or when they feel excited, etc. During fast playback at, say $\alpha=0.5$, the original slow speech sounds fine, but the original fast speech can become too fast resulting in largely reduced intelligibility.

4. there can be long pauses in recorded speech. Even with fast playback at, say $\alpha=0.5$, the pauses can still be undesirably long for previewing purpose.

5. people speak with variable intensity, sometimes loud and sometimes soft. During fast playback, the soft speech is more liable to be missed.

As a result, we propose to do the following:
1. Silent period removal. During a silent period, the speaker is silent for an extended period of time. This should not be confused with pauses between adjacent syllables which carry lexical information. To remove the silent periods, the input signal $x[n]$ is segmented into frames. Energy is computed for each frame. If the frame energy is consistently lower than a threshold for a long period of time, the corresponding frames are removed from the signal. Since many consonant sounds have very weak energy and they are important for intelligibility, a conservatively small threshold is used. Let the resulting signal be $x_{I}[n]$.

2. Syllable segmentation. The $x_{1}[n]$ is further segmented into syllables. This is done by analyzing the energy profile and the first formant frequency $f_{1}$. In [6], it is reported that the midpoint formant frequencies of vowels do not vary as a function of the speaking rate or the vowel duration. For each syllable, there is usually a corresponding local maximum in the energy profile. Sometimes there are multiple local maxima but these can be suppressed due to their close proximity to each other. For some syllables with low energy, the $f_{1}$ is used as auxiliary information to determine the presence of a syllable.

3. Gain equalization. The $x_{1}[n]$ is enhanced in terms of intensity on a syllable-by-syllable basis. The average energy is computed for each syllable. The weakness syllables with low energy are amplified to increase their intelligibility. Let the resulting signal be $x_{2}[n]$.

4. Locally adaptive TSM. As the short syllables in the input signal tend to be extremely short and unintelligible when the input is fast played back, they should be lengthened to increase the intelligibility. In comparison, the long syllables in the input signal appear to be much clearer. This is achieved by assigning larger TSM factors to short syllables and smaller TSM factors to long syllables.

Let $T_{k}$ be the duration of $k^{t h}$ syllable in $x_{2}[n]$. Let $\tilde{T}_{k}$ be the duration of the time scaled version of the $k^{\text {th }}$ syllable. Then the TSM factor $\alpha_{k}$ for the $k^{\text {th }}$ syllable is $\alpha_{k}=\tilde{T}_{k} / T_{k}$. Applying a constraint that all the syllables are to have equal duration in the time scaled output, we get 


$$
\begin{gathered}
\tilde{T}_{k}=\frac{1}{M}\left(\alpha_{o} \sum_{k=1}^{M} T_{k}\right) \\
\alpha_{k}=\frac{\alpha_{o}}{M T_{k}} \sum_{k=1}^{M} T_{k}
\end{gathered}
$$

where $M$ is the number of syllable in the input. Let $P_{k}$ be the $k^{\text {th }}$ pause. The duration of the time scaled version of the $k^{\text {th }}$ pause is $\alpha_{o} P_{\dot{k}}$. This way, the overall time scale modification factor is still $\alpha_{o}$ but all the syllables are equalized to have the same duration.

If the input speech signal is very long (e.g. 1 hour) in the case of a lecture, the computation and memory requirement for the syllable segmentation can be very long. The input can be segmented into short segments (of say 2 minute in duration) and each segment can be processed independently.

\section{RESULTS AND DISCUSSIONS}

The proposed algorithm was tested on many recorded lecture speech files. Fig. 1 shows a typical sentence with and without silent period removal and gain equalization.

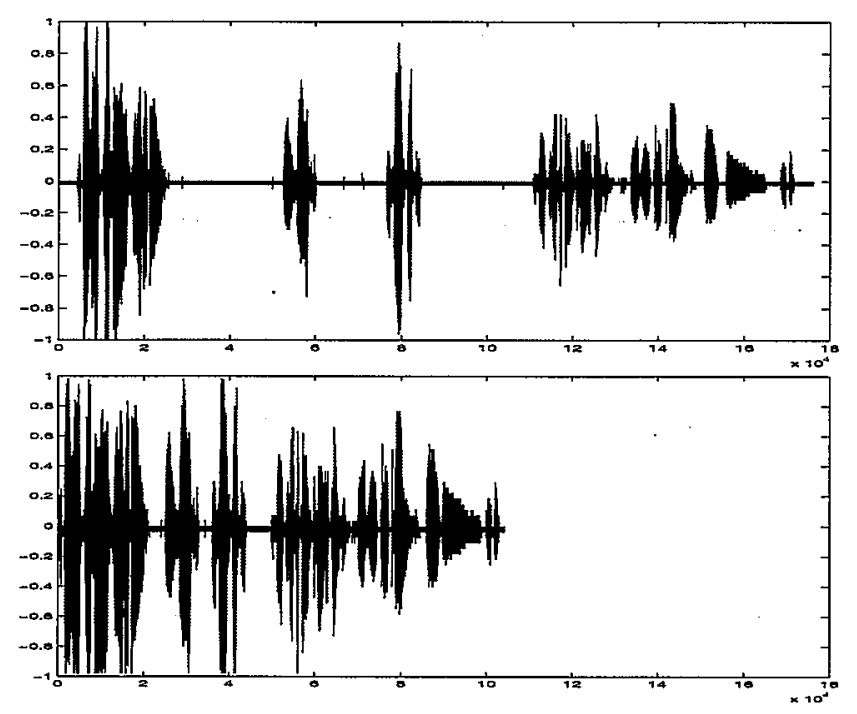

Fig. I Input with (top) and without (bottom) silent period removal and gain equalization.

Note that silent period removal reduces the total duration significantly, by about $40 \%$ in Fig. 1 . The sentence with silent period removed is shown again in Fig. 2 together with its spectrogram. Note that different syllables can have very different duration. Fig. 3 shows the sentence time scale modified with $\alpha_{o}=0.5$. Two cases are shown, one produced by simple TSM and the other by the proposed algorithm. The time varying TSM factor is shown in Fig. 2.

For $\alpha_{o}$ close to unity, simple TSM and the proposed algorithm have similar performance. When the TSM factor $\alpha_{o}$ decreases, the short syllables become increasingly short making it very difficult to comprehend though the longer syllables are relatively more intelligible than the short syllables. When the proposed algorithm is applied, the short syllables are lengthened considerably in time at the expense of having the longer syllables shortened, resulting in significantly improved intelligibility. The gain normalization helps to make the soft syllables more intelligibility. This shows that the proposed algorithm is indeed suitable for fast playback of speech material in distant learning and digital library applications.

\section{CONCLUSION}

In this paper, we propose a novel way for fast playback of speech material with enhanced intelligibility for preview purpose in distance learning, digital library and internet applications. Fast playback is achieved by time scale modification(TSM). Enhanced intelligibility is achieved by silent period removal, gain equalization, and locally adaptive TSM. Simulation results show that the proposed algorithm can increase the intelligibility of the fast playbacked speech significantly.

\section{ACKNOWLEDGEMENT}

This work was supported by RGC Grant \# HKUST 6009/ 97E.

\section{Reference}

[1] D.W. Griffin, J.S. Lim, "Signal Estimation from Modified Short Time Fourier Transform", IEEE Trans. Acoustics, Speech, Signal Processing, Vol 32, pp. 236-242, Feb. 1984.

[2] M.R. Portnoff, "Time Scale Modification of Speech based on Short Time Fourier Analysis", IEEE Trans. Acoustics, Speech, Signal Processing, Vol. 29, pp. 374-390, Jun. 1981.

[3] D. Malah, "Time Domain Algorithms for Harmonic Bandwidth Reduction and Time Scaling of Speech Signals", IEEE Trans. Acoustics, Speech, Signal Processing, Vol. 27, pp. 121-133, Apr. 1979.

[4] S. Roucos, A.W. Wilgus, "High Quality Time Scale Modification for Speech", Proc. of IEEE Int. Conf. Acoustics, Speech, Signal Processing, pp. 493-496, Mar. 1985.

[5] J.W.C. Wong, O.C. Au, P.H.W.Wong, "Fast Time Scale Modification using Envelop-Matching (EM-TSM)", to appear in Proc. of IEEE Int. Sym. Circuits and Systems, May 1998.

[6] T. Gay, "Effect of Speaking Rate on Vowel Formant Movement", J. Acoustic Soc. Am., Vol. 63, No. 1, pp. 223-230,Jan 1978. 

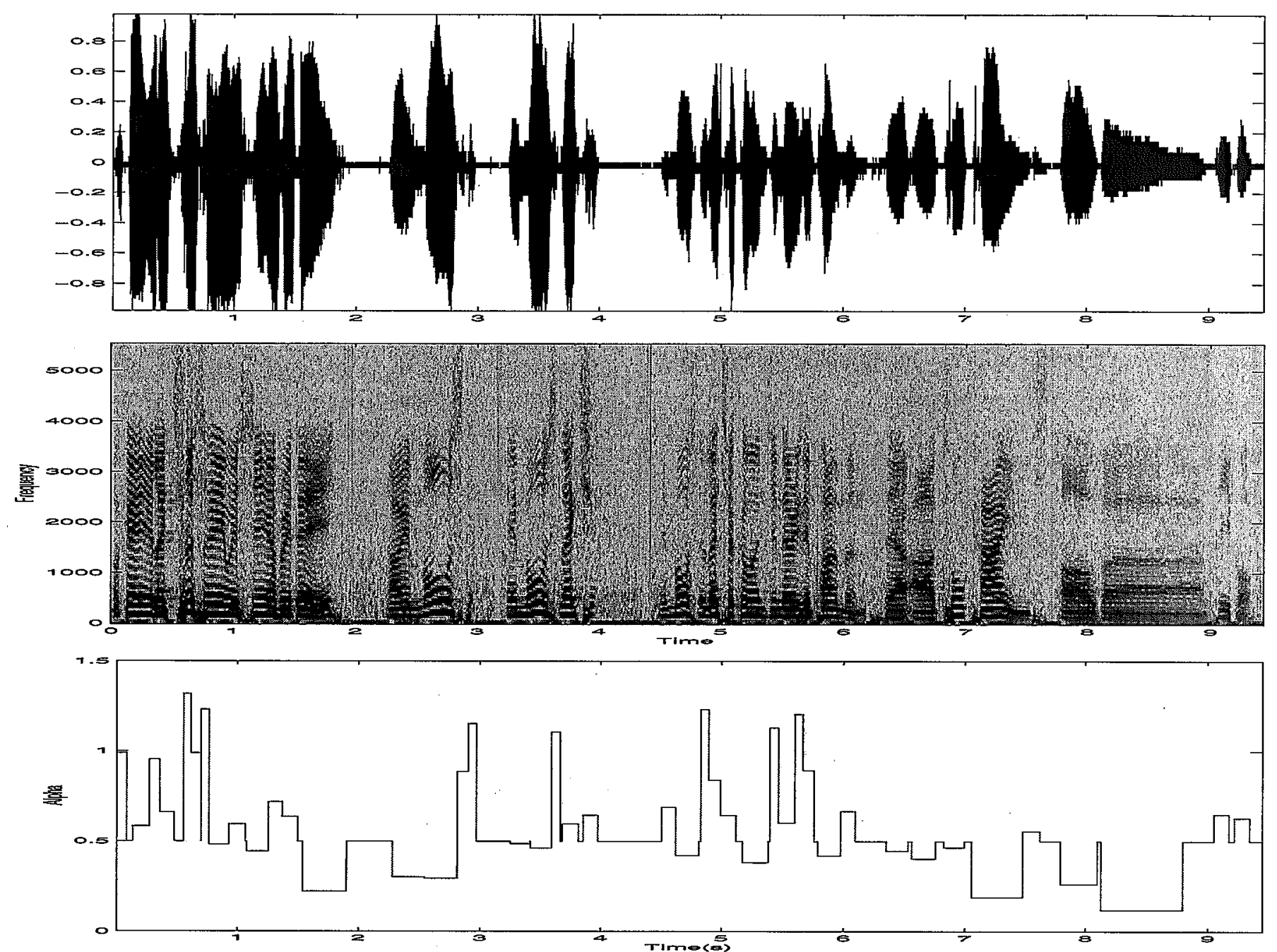

Fig. 2 Top: Input waveform. Middle: Input spectrogram. Bottom: Time varying TSM factor applied to input.
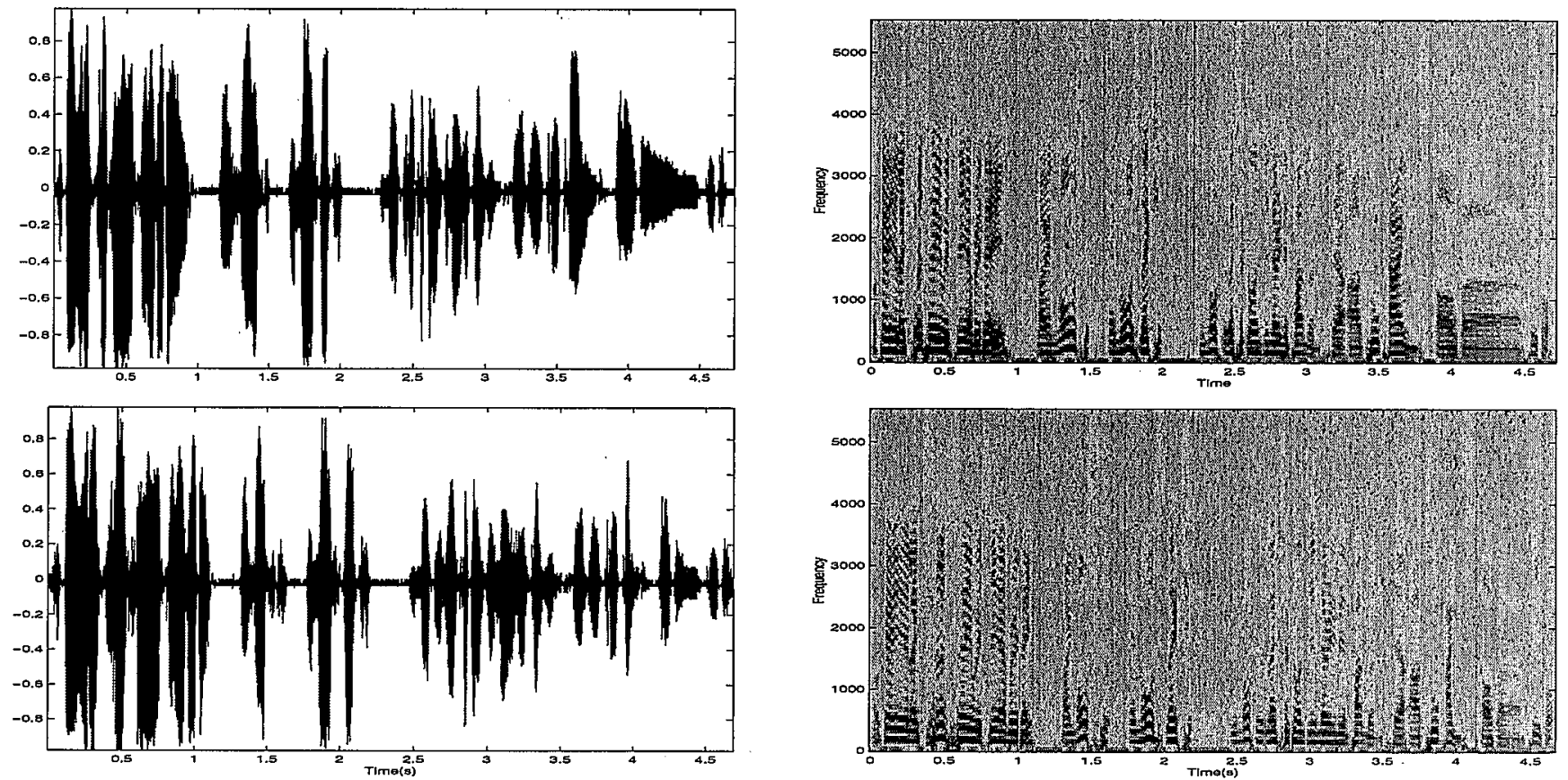

Fig. 3 Top: Input time scaled with simple TSM. Bottom: Input time scaled with proposed adaptive TSM. $\left(\alpha_{0}=0.5\right)$ 